\title{
Repeated whole-lung lavage for unremitting pulmonary alveolar proteinosis: a eight-year follow-up of a case
}

\author{
F. Gao, G.C. Lu, X.Y. Zhou, Z. Yu, H.M. Wang and T. Bian \\ Department of Respiratory Medicine, \\ Wuxi People's Hospital Affiliated to Nanjing Medical University, \\ Jiangsu, China \\ Correspondence author: T. Bian \\ E-mail: taobiancn@126.com
}

Genet. Mol. Res. 13 (3): 6135-6141 (2014)

Received July 30, 2013

Accepted December 5, 2013

Published August 7, 2014

DOI http://dx.doi.org/10.4238/2014.August.7.29

\begin{abstract}
Pulmonary alveolar proteinosis (PAP) is a rare clinical syndrome that was first described in 1958. To date, whole-lung lavage (WLL) is still the gold-standard therapy for PAP. Herein, we report the case of a male patient who was diagnosed with PAP by open-lung biopsy 8 years prior to presentation at our clinic. The man underwent his first WLL in 2004 and showed marked clinical and radiological improvement after the operation. However, after his original presentation, proteinaceous material continued to accumulate in his lungs. Lavage was performed four additional times, but these attempts failed to arrest the decline in pulmonary function. Each lavage resulted in significant, although transient, clinical improvement.
\end{abstract}

Key words: Pulmonary alveolar proteinosis; Whole-lung lavage; Follow-up; Case report 


\section{INTRODUCTION}

Pulmonary alveolar proteinosis (PAP) is a diffuse pulmonary disease characterized by the intra-alveolar accumulation of surfactant lipids and proteins, which impairs pulmonary gas transfer. The severity of this condition ranges from an asymptomatic clinical presentation to respiratory failure and death (Campo et al., 2013). PAP is an extremely rare disorder, occurring worldwide with an estimated prevalence of 0.1 per 100,000 individuals (Trapnell et al., 2003).

Although the pathogenesis of PAP has remained unknown, most investigators consider the condition to be caused by the impaired clearance of lipids and surfactant proteins from the air spaces. These functions are known to be performed by alveolar macrophages and type 2 epithelial cells. In spite of major advances achieved in the late 1990s when Kitamura et al. (1999) and Tanaka et al. (1999) first described the presence of autoantibodies neutralizing granulocyte-macrophage colony-stimulating factor (GM-CSF) in the serum and lung tissue of patients with idiopathic PAP, several specific treatments have been attempted or postulated (Luisetti et al., 2010), but the standard of care remains whole-lung lavage (WLL). The technique has been modified since its original description (Ramirez et al., 1963). In this report, we have presented a case with PAP who underwent WLL four times. This study was conducted in accordance with the declaration of Helsinki and with the approval of the Ethics Committee of Nanjing Medical University. Written informed consent was obtained from all participants. We analyzed baseline functional and biochemical parameters to optimize the patient's long-term follow-up, especially with respect to the need for WLL.

\section{CASE INFORMATION}

A 40-year-old man who had suffered from a cough with sputum for more than 2 years, with no associated fever, presented at our institution. He had previously been seen at another hospital, where he had been diagnosed with pneumonia, and treated unsuccessfully with antibiotics. Upon presentation to our clinic, the patient complained of trouble breathing, so viral infection was suspected. However, the results of laboratory testing were negative. His past medical history showed that he had a chronic history of cough, easy fatigability and shortness of breath upon mild exertion. There was no history of drug intake or exposure to toxic fumes or inorganic dust. The patient had smoked for 15 years, 20 cigarettes per day. He had no particular family history. Further investigations were performed including computed tomography imaging of the chest, which revealed bilateral diffuse reticulonodular opacities and a "crazy paving" pattern suggestive of alveolar proteinosis. After the patient was admitted, open-lung biopsy was performed to confirm the diagnosis. The histopathologic examination revealed eosinophilic secretions with a granular appearance suggestive of PAP (Figure 1A and B).

In 2004, the patient's trouble with breathing worsened, and he presented once more to our institution. Upon arrival, he was conscious on oxygen (oxygen saturation 97\% on 2-3 $\mathrm{L} \mathrm{O}_{2}$ ), pulse rate, 96 beats/min; blood pressure, $115 / 60 \mathrm{mmHg}$; respiratory rate, $24 / \mathrm{min}$; and body temperature, $36.8^{\circ} \mathrm{C}$. The results of biochemical analysis were all within normal ranges. Arterial blood gases on room air were: $69.0 \mathrm{mmHg} \mathrm{PaO}_{2}, 32.2 \mathrm{mmHg} \mathrm{PaCO}_{2}, \mathrm{pH} 7.43$ and $2.4 \mathrm{mM} \mathrm{HCO}_{3}$. Pulmonary function tests were performed and showed a restrictive pattern. WLL was recommended to improve the patient's symptoms. On examination, the patient was 
in respiratory distress and $\mathrm{SaO}_{2}$ at rest on air was $82 \%$. With $3 \mathrm{~L}$ oxygen supplementation via nasal cannula, this value rose to $91 \%$. Examination of the patient's respiratory system revealed bilateral Velcro crackles. The results of examinations of all other systems were unremarkable. He had no other comorbidity.

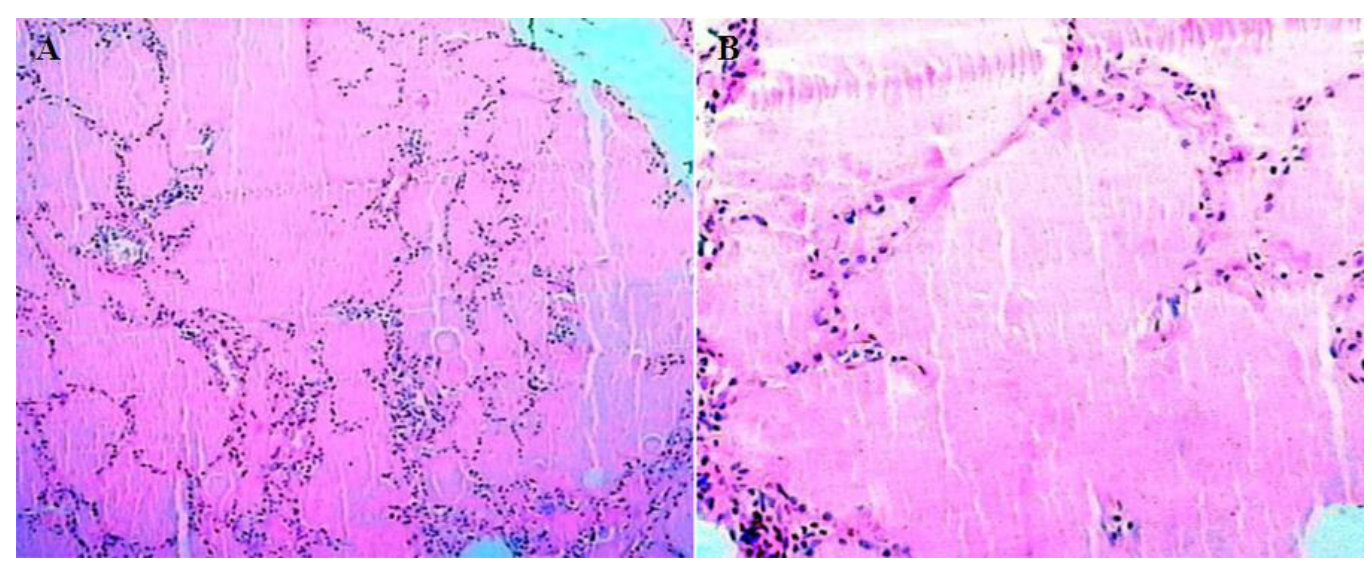

Figure 1. A. Low-power photomicrograph showing alveoli filled with dense, pink proteinaceous material [hematoxylin and eosin (H\&E), 40X]. B. Higher magnification showing macrophages within the proteinaceous deposit (H\&E, 100X).

\section{WLL}

Six days post-admission, left-sided WLL was performed under general anesthesia. Cardiopulmonary monitoring was conducted to ensure that the procedure was smooth and uneventful. After the patient was intubated with a double-lumen endotracheal tube, flexible bronchoscopy was performed to confirm appropriate tube placement. Both the bronchial and the tracheal balloons were inflated to isolate the lungs, and intermittent positive pressure ventilation was initiated with tidal volume of $300-400 \mathrm{~mL} / \mathrm{kg}$ at a rate of 13 breaths $/ \mathrm{min}$. The patient was then placed in the right lateral position with his right side down. Before starting the lavage process, about $200 \mathrm{~mL}$ saline was instilled to fill the lung to its functional residual capacity. Aliquots of 350-450 mL buffered normal saline were then warmed to body temperature and instilled into the lung. The fluid was allowed to flow into the lungs by gravitational force, which was facilitated by hanging the saline bags about two feet above the table surface. After each instillation, the fluid was drained passively by re-positioning the operation table. The initial drainage fluid was milky and opalescent; the procedure was repeated until the effluent appeared to be clear. A total of $14 \mathrm{~L}$ saline was used for lavage of the left lung. Following the procedure, the trachea was extubated and the patient returned to the ward after palinesthesia. One week later, the patient underwent right-sided WLL with the same anesthesia and ventilation techniques. This time, $9.5 \mathrm{~L}$ warmed buffered normal saline was necessary before the effluent became clear (Figure 2A and B). The patient exhibited marked ventilatory and radiological improvement after sequential WLL (Figure 3A-F), with no complications during or after WLL. 


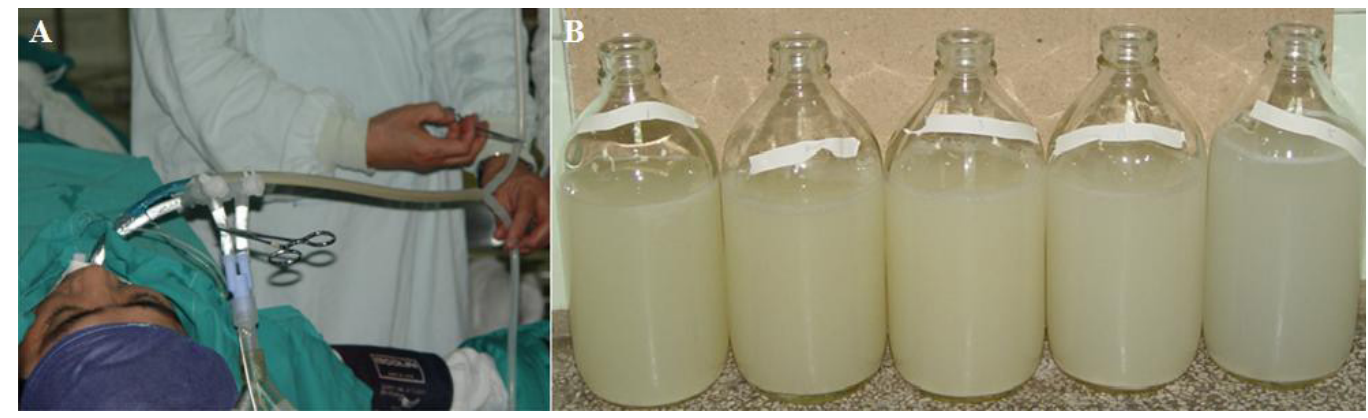

Figure 2. A. Patient undergoing the whole-lung lavage. B. Macroscopic appearance of drainage fluid.

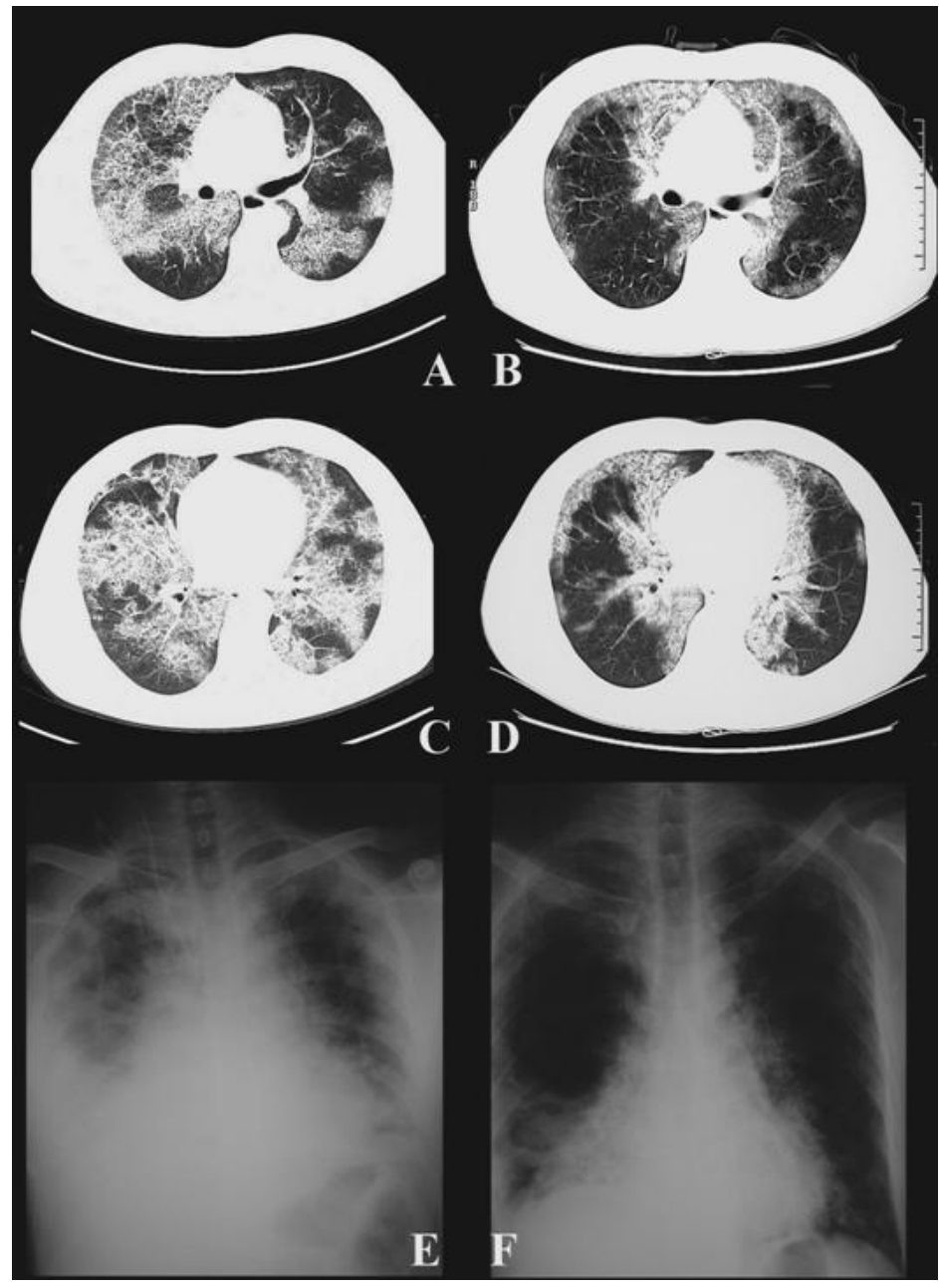

Figure 3. A. and C. Computed tomography (CT) before whole-lung lavage (WLL). B. and D. CT after both sides of WLL. C. Chest X-ray before WLL. D. Chest X-ray after WLL. 


\section{OUTCOME AND FOLLOW-UP}

The patient made a satisfactory recovery and was weaned off oxygen and discharged. After his original presentation, however, this man suffered from several recurrences of proteinaceous material in the lungs. He complained of cyanosis and was finding it more and more difficult to breathe, with an exercise tolerance of less than one flight of stairs. Chest radiographs taken at the time of admission were similar to those seen at the time of the initial presentation. Altogether, four lavages were performed after the patient's diagnosis with PAP. Although the patient recovered satisfactorily each time (Table 1), WLL was not able to prevent this decline in pulmonary function (Figure 4).

\begin{tabular}{|c|c|c|c|c|c|c|}
\hline Time & & $\mathrm{PaO}_{2}(\mathrm{mmHg})$ & $\mathrm{PaCO}_{2}(\mathrm{mmHg})$ & $\mathrm{SaO}_{2}(\%)$ & $\mathrm{HCO}_{3}^{-}(\mathrm{mEq} / \mathrm{L})$ & $\mathrm{pH}$ \\
\hline \multirow[t]{2}{*}{2004} & Before WLL & 69.0 & 32.2 & 92.0 & 16.5 & 7.32 \\
\hline & One week post-WLL & 79.0 & 25.8 & 96.0 & 15.9 & 7.39 \\
\hline \multirow[t]{2}{*}{2006} & Before WLL & 60.2 & 46.2 & 91.7 & 28.0 & 7.39 \\
\hline & One week post-WLL & 63.2 & 42.6 & 90.7 & 26.3 & 7.40 \\
\hline \multirow[t]{2}{*}{2009} & Before WLL & 53.0 & 28.0 & 88.0 & 18.0 & 7.42 \\
\hline & One week post-WLL & 60.0 & 41.0 & 89.0 & 22.0 & 7.35 \\
\hline \multirow{2}{*}{2011} & Before WLL & 52.5 & 26.0 & 88.0 & 16.3 & 7.35 \\
\hline & One week post-WLL & 65.3 & 32.3 & 91.0 & 17.6 & 7.36 \\
\hline
\end{tabular}

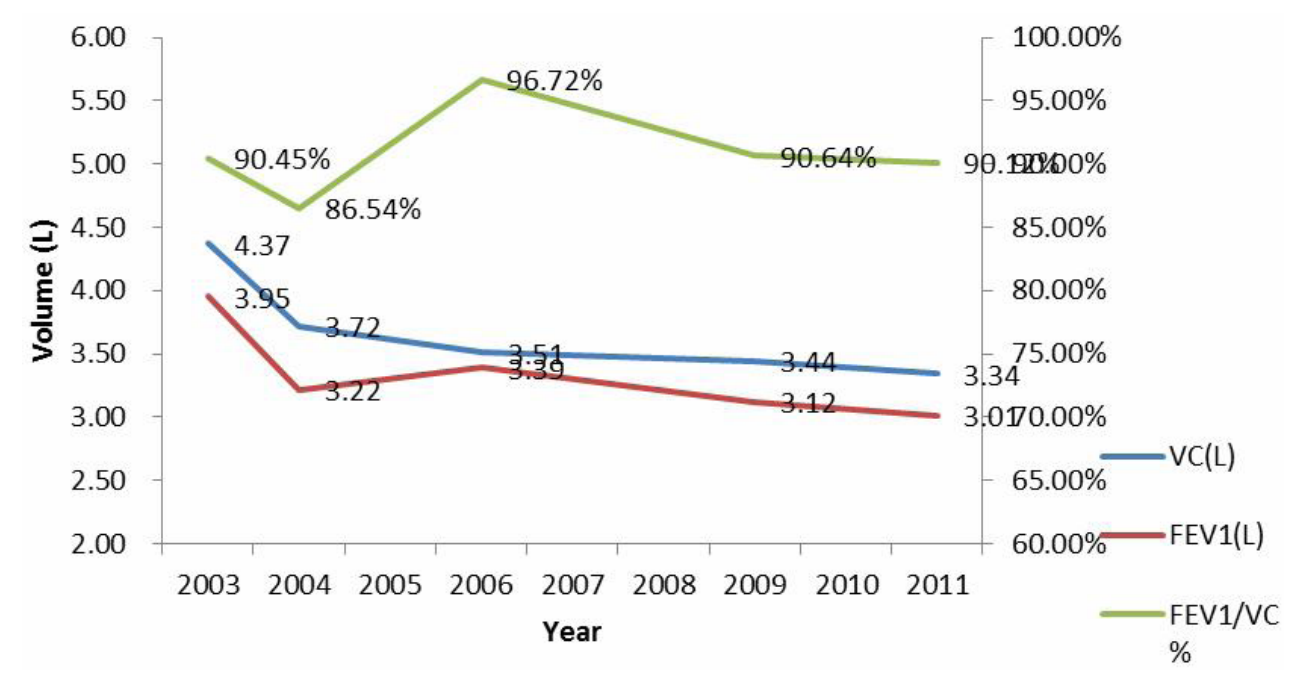

Figure 4. Follow-up lung function.

\section{DISCUSSION}

PAP is a rare disorder characterized by the accumulation of lipoproteinaceous substance in the bronchoalveolar tree. Three distinct subgroups of the disease are currently recognized: congenital, idiopathic (primary), and secondary. More than $90 \%$ of all reported cases 
are classified as the autoimmune type, which is associated with the presence of GM-CSF autoantibodies.

PAP has a variable clinical course ranging from spontaneous resolution to death with pneumonia or respiratory failure. Previous studies suggested that less than one-third of the patients showed apparently spontaneous resolution after an unspecified period of observation (Tanaka et al., 1999). Another subset of patients follows a chronic but stable clinical course. In most patients, the disease is slowly progressive, ultimately ending in respiratory failure. In a small percentage of patients, it runs a rapid downhill course and death is imminent without urgent treatment.

Although increasing evidence indicates that GM-CSF therapy may be beneficial for patients with PAP, the success rate for GM-CSF is so far not sufficient to displace the success of WLL therapy (Bonella et al., 2011). As emphasized above, WLL is the current standard of care for autoimmune and other forms of PAP (Huizar and Kavuru, 2009; Michaud et al., 2009; Luisetti et al., 2010). In the pre-WLL era, death occurred mostly because of progressive respiratory failure and, to a lesser extent, superimposed respiratory opportunistic infections. As shown in Figure 2A and B, the WLL procedure was undertaken to address PAP recurrences. The patient was asked for repeated lavages every 2-3 years according to the presence of persistent or progressive respiratory failure. Following WLL, symptoms, oxygenation, and chest radiographs showed that the patient improved over the next few weeks (Figure 3A-F and Table 1). In the present case, the average improvement in $\mathrm{PaO}_{2}$ levels after WLL was 3-13 mmHg (overall mean $8.25 \mathrm{mmHg}$ ) within the first week (Table 1). The median duration of clinical benefit following WLL was 15-20 months. However, repeated WLL was not able to prevent PAP relapses and the associated impairment of pulmonary function (Figure 4).

In general, WLL is well tolerated. The major potential complication is intraoperative refractory hypotension, which tends to be more common while the first lung is being lavaged. Hyperbaric oxygen, cardiopulmonary bypass, and temporary venovenous extracorporeal gas exchange have all been used in the past, but have not been considered necessary in more recent studies (Beccaria et al., 2004). In addition, it has been reported that $70 \%$ of patients followed beyond 3 years remain free of recurrent PAP manifestations (Beccaria et al., 2004). Because infection is another significant factor influencing the outcomes of PAP patients (Cai et al., 2004), a life-time regular outpatient follow-up program for the timely diagnosis and treatment of recurrence as well as the treatment of pulmonary infections could play a vital and essential role in determining patient outcomes after WLL. However, the PAP patient presented here did not cooperate with the planning of regular visits. Nonetheless, the results of long-term follow-up showed that the procedure was potentially safe and efficacious with long-lasting benefits (15-20 months). The patient showed symptomatic, radiographic and functional improvement after WLL.

Our findings show that repeated WLL alleviated clinical symptoms, reduced hypoxia and improved the patient's quality of life. In addition, our report demonstrated that WLL could not stop the progression of the disease, but did slow down the decline in pulmonary function. Further studies will be necessary to develop novel approaches for the treatment of PAP.

\section{REFERENCES}

Beccaria M, Luisetti M, Rodi G, Corsico A, et al. (2004). Long-term durable benefit after whole lung lavage in pulmonary alveolar proteinosis. Eur. Respir. J. 23: 526-531.

Bonella F, Bauer PC, Griese M, Ohshimo S, et al. (2011). Pulmonary alveolar proteinosis: new insights from a singlecenter cohort of 70 patients. Respir. Med. 105: 1908-1916. 
Cai HR, Cui SY, Jin L, Huang YZ, et al. (2004). Pulmonary alveolar proteinosis treated with whole-lung lavage utilizing extracorporeal membrane oxygenation: a case report and review of literatures. Chin. Med. J. 117: 1746-1749.

Campo I, Mariani F, Rodi G, Paracchini E, et al. (2013). Assessment and management of pulmonary alveolar proteinosis in a reference center. Orphanet. J. Rare Dis. 8: 40.

Huizar I and Kavuru MS (2009). Alveolar proteinosis syndrome: pathogenesis, diagnosis, and management. Curr. Opin. Pulm. Med. 15: 491-498.

Kitamura T, Tanaka N, Watanabe J, Uchida, et al. (1999). Idiopathic pulmonary alveolar proteinosis as an autoimmune disease with neutralizing antibody against granulocyte/macrophage colony-stimulating factor. J. Exp. Med. 190: 875-880.

Luisetti M, Kadija Z, Mariani F, Rodi G, et al. (2010). Therapy options in pulmonary alveolar proteinosis. Ther. Adv Respir. Dis. 4: 239-248.

Michaud G, Reddy C and Ernst A (2009). Whole-lung lavage for pulmonary alveolar proteinosis. Chest 136: 1678-1681.

Ramirez J, Schultz RB and Dutton RE (1963). Pulmonary alveolar proteinosis: a new technique and rationale for treatment. Arch. Intern. Med. 112: 419-431.

Tanaka N, Watanabe J, Kitamura T, Yamada Y, et al. (1999). Lungs of patients with idiopathic pulmonary alveolar proteinosis express a factor which neutralizes granulocyte-macrophage colony stimulating factor. FEBS Lett. 442: 246-250.

Trapnell BC, Whitsett JA and Nakata K (2003). Pulmonary alveolar proteinosis. N. Engl. J. Med. 349: 2527-2539. 